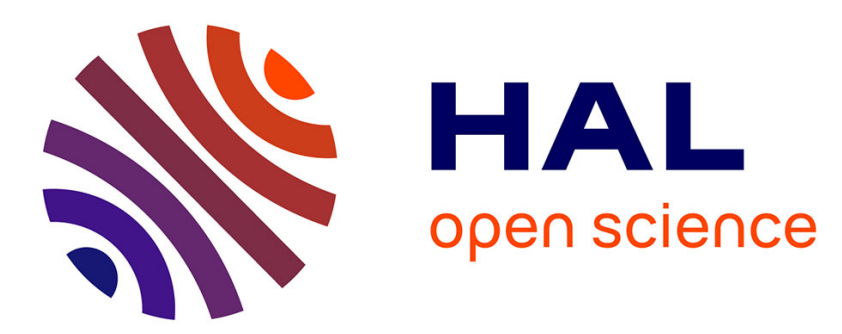

\title{
Numerical and experimental analysis of the correlation between EIT data eigenvalues and two-phase flow phase fraction
}

C Dang, M Darnajou, C. Bellis, G Ricciardi, H. Schmidt, S. Bourennane

\section{- To cite this version:}

C Dang, M Darnajou, C. Bellis, G Ricciardi, H. Schmidt, et al.. Numerical and experimental analysis of the correlation between EIT data eigenvalues and two-phase flow phase fraction. Measurement Science and Technology, 2020, 31 (1), pp.015302. 10.1088/1361-6501/ab3d63 . hal-02357608

\section{HAL Id: hal-02357608 \\ https://hal.science/hal-02357608}

Submitted on 14 Nov 2019

HAL is a multi-disciplinary open access archive for the deposit and dissemination of scientific research documents, whether they are published or not. The documents may come from teaching and research institutions in France or abroad, or from public or private research centers.
L'archive ouverte pluridisciplinaire HAL, est destinée au dépôt et à la diffusion de documents scientifiques de niveau recherche, publiés ou non, émanant des établissements d'enseignement et de recherche français ou étrangers, des laboratoires publics ou privés. 


\title{
Numerical and Experimental Analysis of the Correlation Between EIT Data Eigenvalues and Two-phase Flow Phase Fraction
}

\author{
C. Dang ${ }^{1,2}$, M. Darnajou ${ }^{1,2}$, C. Bellis ${ }^{3}$, G. Ricciardi ${ }^{1}$, H. Schmidt ${ }^{4}$, and \\ S. Bourennane ${ }^{2}$ \\ 1: DEN/DTN/STCP/LTHC, CEA Cadarache, FRANCE \\ 2: Institut Fresnel, CNRS, Centrale Marseille, Aix Marseille Univ, FRANCE \\ 3: Aix Marseille Univ, CNRS, Centrale Marseille, LMA, FRANCE \\ 4: Erlangen Center, Framatome GmbH, GERMANY \\ (Email: dang.chunhui@centrale-marseille.fr)
}

\section{ABSTRACT}

Electrical Impedance Tomography (EIT) is an imaging technique with advantages of nonintrusiveness, low-cost and high temporal resolution, which is promising for multiphase flow instrumentation. However, it produces smooth images with low spatial resolution where the interface between phases cannot be distinguished and from which the phase fraction cannot be estimated correctly. In this article, an eigenvalue analysis of EIT raw data is used to estimate the void fraction, i.e. the phase area ratio in $2 \mathrm{D}$, without reconstructing images. For a given EIT sensor, each acquisition frame is represented by an impedance matrix whose eigenvalues are computed after normalization. The main characteristics of the eigenvalue distribution for different two-phase flow patterns within a cylindrical pipe are analyzed numerically. The behaviors of the leading eigenvalue and of the sum of the absolute values of the following ones are assessed as functions of the void fraction. This leads to an estimation of the two-phase flow void fraction based on the characteristics of the EIT sensor configuration. The presented numerical results highlight the existing correlation between the eigenvalues and the void fraction for the phase distribution patterns considered. These simulation results are compared with experimental static tests for validation.

\section{KEYWORDS}

Electrical Impedance Tomography, impedance matrix, flow patterns, static test.

\section{Introduction}

In the research field of experimental multiphase flow, instrumentation techniques for local void fraction (phase area ratio) and phase distribution estimation are in great need. These parameters are essential to understand the heat transfer coefficient and assess the risk of critical boiling, for example in nuclear industry [1,2]. Various techniques have been developed for multiphase flow phase fraction estimation, for example, wire-mesh sensors can provide information about local, cross-section or in-situ volume profiles and distributions of phase fraction, but they have disruptive effects on the flow [3]; optical probes are sensitive to 
interfacial passages enabling to measure local void fraction with a high precision, however, they are intrusive [4], as are electrical probes as well [5]; X-ray and Gamma-ray tomography allow fast measurements of multiphase flows at high spatial resolution but they require high acceleration voltage (hundreds of $\mathrm{kV}$ ) and radiation protection [6, 7]; Electrical Capacitance Tomography (ECT) has similar characteristics to the EIT but it requires high voltage excitation signals. Note that Huang et al. [8] used ECT to measure void fraction based on image reconstruction with a satisfactory accuracy. With the advantages of non-intrusiveness, high temporal resolution and low cost, the Electrical Impedance Tomography (EIT) technique seems to be promising for dynamic multiphase flow instrumentation.

EIT is a technique to determine the material distribution inside a $2 \mathrm{D}$ or $3 \mathrm{D}$ domain based on their constitutive electric properties, e.g. the electric admittivity (conductivity and permittivity), with an image acquisition rate that can be up to hundreds of frames per second [9]. For an EIT sensor, the measurements are acquired on the boundary and the typical way to handle the measurement data is through image reconstruction, which leads to an approximate reconstruction of the admittivity distribution within the domain. Theoretically, continuous boundary measurement determines a unique solution to the reconstruction problem [10], while in practice the boundary measurement is limited by the number of electrodes.

A number of reconstruction methods have been proposed based on non-iterative or iterative algorithms. Some non-iterative algorithms are based on linear approximation, such as the linear back projection method [11], the one-step Newton method [12,13] or the Calderon's approach [14]. Iterative methods tackle the reconstruction problem by minimizing some cost functions, e.g. the variational method [15] or the modified Newton-Raphson method [16]. In general, non-iterative algorithms produce smooth images with low spatial resolution compared to the hard-field tomography techniques, like X-ray or Gamma-ray tomography [17]. This makes it difficult to distinguish the phase interface in the reconstructed images. Iterative methods yield reconstructions at higher resolutions but they usually require high computation power. In the present article, an alternative methodology is proposed to estimate the void fraction of two-phase flows. It circumvents the need to reconstruct images in the extreme non-linear case of two-phase flows, which are characterized by a high resistivity contrast between phases, while it allows to consider a wide range of void fraction.

A typical EIT sensor has a set of electrodes installed on the boundary of a domain, Figure 1 shows an EIT sensor with 16 electrodes. A stimulation current (or voltage) are exerted on one pair of selected electrodes (noted as source and drain electrode), the corresponding voltage (or current) at all the other electrodes are measured, this is called a stimulation pattern. This procedure continues until each independent pair of electrodes has been selected as source and drain [18,19], a strategy referred to as the full scan stimulation strategy. Since source and drain are essentially the same, the measurements would be reciprocal for a specific pair of electrodes. For an EIT sensor with $\ell$ electrodes, there are $\ell(\ell-1) / 2$ independent stimulation patterns in total for one frame of image. Each stimulation pattern has a representative bulk impedance, which depends on the phase area ratio and distribution, as well as on the source/drain electrodes. The impedance array of each frame can be conveniently arranged into a $\ell \times \ell$ symmetric matrix, whose diagonal entries are related to the mutual impedances. In the work of Fang et al. [20], the relation between the void fraction and the eigenvalues of the capacitance matrix obtained from ECT data is investigated and 
it is shown that the multiplicity of the eigenvalues is correlated to the degree of symmetry of the flow. Dupré et al. applied the eigenvalue analysis to ECT data for the purpose of flow regime identification as well as the estimation of two-phase flow void fraction, the result is promising [19, 21]. In this context, and up to the Author's knowledge, the literature dealing with void fraction estimation from EIT data and through eigenvalue analysis is scarce. Therefore, in the present article, the impedance matrix is investigated and its eigenvalues are used to devise some metrics of the void fraction.

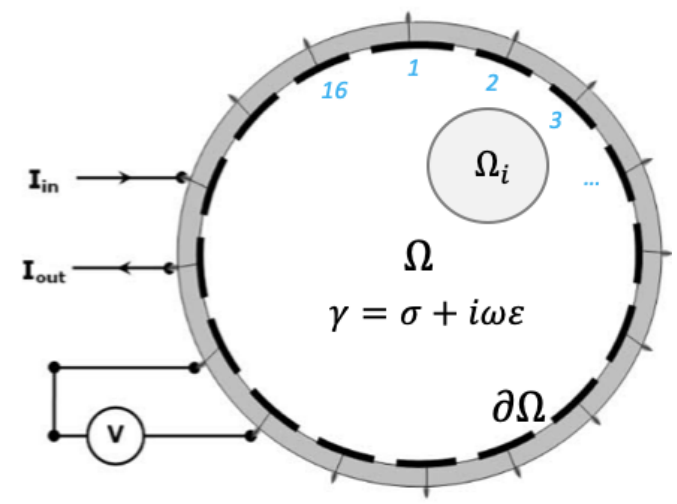

Figure 1. schematic of an EIT sensor with 16 electrodes.

The EIT problem is associated with an elliptic boundary-value problem and it aims at recovering the information on the admittivity distribution inside a domain of interest from boundary measurements. In Section 2, the mathematical basis of EIT is introduced with the Neumann-to-Dirichlet (NtD) operator along with the bulk impedance that is the discrete realization of the former. The impedance data can be computed numerically using the Finite Element Method (FEM) and arranged into a square impedance matrix, the eigenvalues of which are the focus of this study. In Section 3, the typical regimes of two-phase flows are classified into three different canonical cases that are studied separately: the distribution of the eigenvalues of the impedance matrix is studied for each pattern based on a suitable normalization, with the relation between eigenvalues and void fraction being investigated numerically. In Section 4, the influence of noisy data and an extension to conductive inclusions are investigated. In Section 5, the EIT system developed in the laboratory is introduced and the simulation results are validated by experimental results associated with static configurations. These results are discussed and concluded in Section 6.

\section{Mathematical basis}

Considering the electric field in a two- or three-dimensional domain $\Omega$, from Maxwell's equation, the electric potential $u$ inside is governed by,

$$
\nabla \cdot(\gamma(x) \boldsymbol{\nabla} u(x))=0, \quad x \text { in } \Omega,
$$

where $\gamma(x)=\sigma(x)+i \omega \epsilon(x)$ is the isotropic admitivity distribution in $\Omega$, in which $\sigma$ is the electric conductivity, $\epsilon$ is the electric permittivity, $\omega$ is the stimulation frequency. In the 
application to multiphase flow instrumentation, usually only the conductivity $\sigma$ is considered, because the electric permittivity $\epsilon$ of liquid phase can be neglected in the chosen working frequency range of EIT sensors [22].

\subsection{Neumann-to-Dirichlet map}

The domain is assumed to be homogeneous except for a number of non-conductive inclusions, which are denoted as $\Omega_{\mathrm{i}}$. These inclusions have a conductivity significantly different with the background, and they are simply connected domains contained in $\Omega$. Specifically for two-phase flow, the conductivity $\sigma$ of the water phase is within $10^{-4}-10^{-2} \mathrm{~S} / \mathrm{m}$, and that of gas is around $10^{-15}-10^{-9} \mathrm{~S} / \mathrm{m}$, i.e.,

$$
\sigma= \begin{cases}\sim 0 & \text { in } \Omega_{\mathrm{i}}, \\ 1 & \text { in } \Omega \backslash \Omega_{\mathrm{i}},\end{cases}
$$

with $\sigma$ of the water phase normalized to 1 .

Denoting as $\boldsymbol{n}$ the unit outward normal vector on the boundary $\partial \Omega$, which is assumed to be smooth, we have the Neumann boundary conditions

$$
\sigma \nabla u \cdot \boldsymbol{n}=f \quad \text { on } \partial \Omega,
$$

in which $f \in L^{2}(\partial \Omega)$ represents the boundary current that satisfies $\int_{\partial \Omega} f \mathrm{~d} S=0$. Note that the model (2) entails that $\sigma \nabla u \cdot \boldsymbol{n} \approx 0$ on the boundary $\partial \Omega_{\text {i }}$ of the inclusions.

Introducing the functional space $H_{\diamond}^{1}(\Omega)=\left\{\varphi \in H^{1}(\Omega): \int_{\partial \Omega} \varphi \mathrm{d} S=0\right\}$, the Neumann boundary value problem is as follows: find $u \in H_{\diamond}^{1}(\Omega)$ that satisfies

$$
\int_{\Omega} \sigma \nabla u \cdot \nabla \varphi \mathrm{d} V=\int_{\partial \Omega} f \varphi \mathrm{d} S, \quad \forall \varphi \in H_{\diamond}^{1}(\Omega) .
$$

On denoting $L_{\diamond}^{2}(\partial \Omega)=\left\{\varphi \in L^{2}(\Omega): \int_{\partial \Omega} \varphi \mathrm{d} S=0\right\}$, the Neumann-to-Dirichlet (NtD) map is introduced as $\Lambda_{\sigma}: L_{\diamond}^{2}(\partial \Omega) \rightarrow L_{\diamond}^{2}(\partial \Omega)$ so that the boundary potential can be written as $\Lambda_{\sigma} f=\left.u\right|_{\partial \Omega}$, where $u \in H_{\diamond}^{1}(\Omega)$ is the solution to (4). The boundary potential can be measured and compared with the boundary potential $\Lambda_{0} f=\left.u_{0}\right|_{\partial \Omega}$ for the same $f$ and $\Omega$ but without inclusions, i.e. $\Omega_{\mathrm{i}}=\emptyset$, with $u_{0} \in H_{\diamond}^{1}(\Omega)$ being the solution of:

$$
\int_{\Omega} \nabla u_{0} \cdot \nabla \varphi \mathrm{d} V=\int_{\partial \Omega} f \varphi \mathrm{d} S, \quad \forall \varphi \in H_{\diamond}^{1}(\Omega),
$$

which corresponds to the reference problem with a homogeneous conductivity distribution inside the domain $\Omega$. The relative NtD map is denoted as $\Pi=\Lambda_{\sigma}-\Lambda_{0}$. In the study by M. Hanke et al. [23], the eigenvalues of $\Lambda_{\sigma}-\Lambda_{0}$ are used to locate the inhomogeneities non-iteratively.

\section{$2.2 \quad$ Electrode models}

In practical implementation, the current density cannot be measured, only the current or voltage at discrete electrodes could be obtained. There are various electrode models available 
depending on their assumptions on current density, i.e. the gap model, the shunt model and the complete model [24,25]. The gap model assumes that the current density is constant over electrodes, while the shunt model considers that the integral of the current density over the electrode equals to the total current flowing through that electrode. Furthermore, the complete model is based on the shunt model, but takes into account the electrochemical effect at the interface between the electrode and the probed medium, which is called the "contact impedance". Compared to the gap model, the shunt and complete models are closer to reality [18]. In the numerical simulation part, the shunt model is used, while in the static tests, the complete model is considered, the electrode specific contact impedance is computed and excluded from the measurement data.

In the shunt model, considering a number $\ell$ of identical electrodes placed on $\partial \Omega$ equally spaced, the integral of the current density over the electrode is equal to the current through this electrode, while the current density at the isolated gaps between electrodes is zero, i.e.

$$
\int_{e_{k}} \sigma \nabla u \cdot \boldsymbol{n} \mathrm{d} s=I_{k} \text { for } k=1, \ldots, \ell \text { while } \sigma \nabla u \cdot \boldsymbol{n}=0 \text { on } \partial \Omega \backslash \bigcup_{k} e_{k},
$$

where $I_{k}$ is the current passing through the $k^{\text {th }}$ electrode and $e_{k}$ is the surface of the $k^{\text {th }}$ electrode. Besides, the electrodes are assumed to be perfectly conducting so that the electrostatic potential $\left.u\right|_{e_{k}}$ is constant at each electrode. Recall that the electrostatic potential inside the domain satisfies the Laplace equation (since $\sigma=1$ in $\Omega \backslash \Omega_{\mathrm{i}}$ ),

$$
\Delta u=0, \quad \text { in } \Omega \backslash \Omega_{\mathrm{i}} .
$$

The shunt model has a unique solution. This allows to define a matrix $\boldsymbol{Z}$, which is the discrete mapping from the boundary current stimulations to the boundary voltage measurements. The matrix $\boldsymbol{Z}$ is referred to as the impedance matrix and it takes over the role of the NtD operator $\Lambda_{\sigma}$ as its discrete representation. Defining the discrete version of $L_{\diamond}^{2}(\partial \Omega)$ as the $\ell$-dimensional vector space $\mathbb{R}_{\diamond}^{\ell}=\left\{y=\left[y_{1} \cdots y_{\ell}\right] \in \mathbb{R}^{\ell}, \sum_{i=1}^{\ell} y_{i}=0\right\}$, one gets

$$
\begin{aligned}
Z: & \mathbb{R}_{\diamond}^{\ell} \rightarrow \mathbb{R}_{\diamond}^{\ell} \\
& {\left[I_{0} I_{1} \cdots I_{\ell}\right] \longmapsto\left[\begin{array}{llll}
V_{0} & V_{1} & \cdots & V_{\ell}
\end{array}\right] }
\end{aligned}
$$

where $V_{k}=\left.u\right|_{e_{k}}$ for $k=1, \ldots, \ell$ are the boundary measurements of the electric potential solution $u$ corresponding to the set of imposed currents $I_{k}$ considered.

Each element in $\boldsymbol{Z}$ is a bulk impedance corresponding to a certain imposed current and boundary measurement. when the continuous liquid phase is the only conducting phase, given the mixture conductivity $\sigma_{m}$, the liquid conductivity $\sigma_{l}$ and the liquid volume fraction $\alpha_{l}$, the Maxwell-Hewitt relation [26] reads:

$$
\alpha_{l}=1-\frac{1-\sigma_{m} / \sigma_{l}}{1+\sigma_{m} / \sigma_{l}}, \text { in } 2 \mathrm{D}, \quad \alpha_{l}=1-\frac{1-\sigma_{m} / \sigma_{l}}{1+0.5 \cdot \sigma_{m} / \sigma_{l}} \text {, in 3D. }
$$

This relation yields an approximation of the volume fraction of each phase, as shown in 27. The water-gas two-phase flow system conforms this relation, so that the measured impedance can be correlated to the void fraction. In the present study, the impedance matrix contains the impedance of all the possible electrode separations. In this framework, the impedance 
matrix is assumed to be directly correlated to the void fraction and our objective is to investigate this correlation based on an eigenvalue analysis of numerical and experimental standardized tests.

\subsection{Impedance matrix and normalization}

For each stimulation pattern, denoting the source and drain electrodes pair as $(i, j)$, there is a corresponding bulk impedance $Z_{i, j}$, which is governed by the conductivity distribution $\sigma(x)$ in $\Omega$, as well as the selected source and drain electrodes. The impedance between source electrode $j$ and drain electrode $i$ is the mutual impedance of $Z_{i, j}$, so that they satisfy the reciprocity relation $Z_{i, j}=Z_{j, i}$. According to the definition of $\mathbb{R}_{\diamond}^{\ell}$, the diagonal term $Z_{i, i}$ is not measured but computed by summing up the corresponding mutual impedances as $Z_{i, i}=-\sum_{j \neq i} Z_{i, j}$, which finally yields the $\ell \times \ell$ square impedance matrix $\boldsymbol{Z}=\left(Z_{i, j}\right)_{1 \leq i, j \leq \ell}$.

The relative NtD map $\Pi$ is commonly used in EIT to retrieve information on the probed medium. This amounts to consider the matrix $\Delta \boldsymbol{Z}=\boldsymbol{Z}-\boldsymbol{Z}^{\mathbf{0}}$, where $\boldsymbol{Z}^{\mathbf{0}}$ denotes the impedance matrix of the homogeneous conductivity distribution for which $\Omega_{\mathrm{i}}=\emptyset$. The matrix $\Delta \boldsymbol{Z}$ is referred to as the Differential Impedance matrix (DIM). To perform a nondimensional analysis, a normalization method of the matrix entries $Z_{i, j}$ is needed to reduce the dependencies on the geometric parameters of the sensor and on the background conductivity, as well as to enhance the presence of inhomogeneities. In this study, the normalization method is proposed as:

$$
\hat{Z}_{i, j}=\frac{Z_{i, j}-Z_{i, j}^{0}}{Z_{i, j}^{0}} \quad \text { for } i, j \in\{1, \ldots, \ell\} .
$$

The associated matrix $\hat{\boldsymbol{Z}}=\left(\hat{Z}_{i, j}\right)_{1 \leq i, j \leq \ell}$ is referred to as the Normalized Impedance Matrix (NIM). Note that $\hat{Z}_{i, j}$ is equal to zero for all $i, j$ when $\Omega_{\mathrm{i}}=\emptyset$ and is infinite when $\Omega_{\mathrm{i}}=\Omega$.

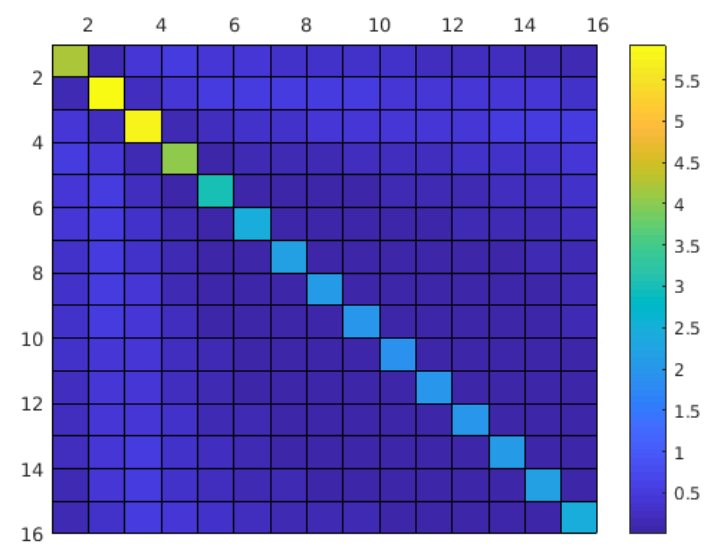

(a) Differential impedance matrix $\Delta \boldsymbol{Z}$

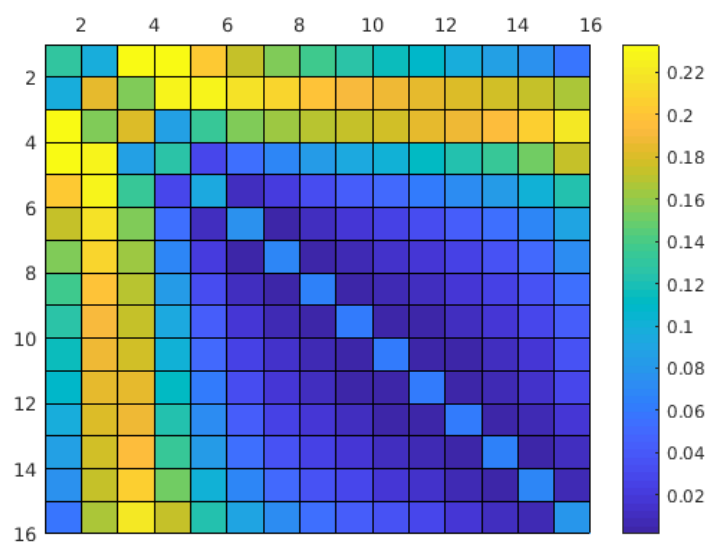

(b) Normalized impedance matrix $\hat{\boldsymbol{Z}}$

Figure 2. Effect of the normalization on the entries of the impedance matrices in the case of a single bubble (Case 1). 
Considering the case shown in Figure 1 with a single bubble of radius 0.3 and an EIT system with 16 electrodes, the corresponding DIM and NIM are visualized with the horizontal axes as the matrix entry indices and the vertical axis for the associated entry value, as in Figure 2, The effect of normalization is highlighted: The DIM has significant values on the diagonal, which is consistent with the computation of its diagonal terms $Z_{i, i}$. The normalization diminishes this effect and enhances the impedance discrepancies between electrodes, see for example the entries associated with the electrodes 2 and 3 that are the closest to the bubble.

\subsection{Eigenvalue analysis}

As, by definition, the normalized impedance matrix $\hat{Z}$ is a real-valued symmetric matrix, it is diagonalizable and its eigenvalues $\lambda^{(i)}$ are real. Therefore, it has a set of $\ell$ pairs of eigenvectors $\boldsymbol{v}^{(i)} \in \mathbb{R}_{\diamond}^{\ell}$ and eigenvalues $\lambda^{(i)} \in \mathbb{R}$ satisfying

$$
\hat{\boldsymbol{Z}} \cdot \boldsymbol{v}^{(i)}=\lambda^{(i)} \boldsymbol{v}^{(i)}, \quad i \in\{1, \ldots, \ell\} .
$$

Sorting the eigenvalues in an increasing order, the resulting eigenvalue array is defined as

$$
\lambda=\left[\begin{array}{llll}
\lambda^{(1)} & \lambda^{(2)} & \cdots & \lambda^{(\ell)}
\end{array}\right] \text { with } \lambda^{(1)}<\lambda^{(2)}<\cdots<\lambda^{(\ell)},
$$

where $\lambda^{(\ell)}$ is the leading eigenvalue. Moreover, considering the rotational symmetry of the electrodes placement, the eigenvalues of $\hat{\boldsymbol{Z}}$ are invariant under similarity transformations of the electrodes, such as rotation or renumbering.

The largest eigenvalues contain the most information about the matrix $\hat{\boldsymbol{Z}}$ and represent its main features, while $\hat{\boldsymbol{Z}}$ is decided by the conductivity distribution within the probed medium, the other smallest eigenvalues are also affected by it but contain qualitatively less information. Hereafter, the characteristics of these eigenvalues are investigated numerically. Note that in the configurations where different numbers of electrodes are used to impose the current and to measure the potential on the domain boundary, then the associated impedance matrices are not square. In such cases, their singular value decompositions can be considered alternatively.

\section{Numerical simulations}

In the previous section, the mathematical model of EIT and the normalized impedance matrix are introduced. In this section, numerical simulations are considered to investigate the relation between the eigenvalues and the conductivity distribution within the probed medium.

\subsection{Canonical cases considered}

The typical regimes of two-phase flows include bubbly flow, stratified flow, slug/plug/churn flow and annular flow, see 28 30]. The phase distributions can be classified into three classes, regardless of pipe placement and gas/liquid flow directions, that is, (i) Single bubble 
case, representing the water slug in slug/plug/churn flow and the annular flow as well; (ii) Stratified case; (iii) Multiple bubbles case, relevant to the bubbly flow and the bubble cloud that follows the slug in slug/plug/churn flow.

Consider a $2 \mathrm{D}$ circular pipeline $\Omega$ with a radius $r_{0}=1$, i.e. $\Omega=\left\{(x, y): x^{2}+y^{2}<1\right\}$. Given the rotational symmetry of the impedance matrix, three classes of bubble distribution patterns are defined as follows:

- Case 1 (single bubble): $\Omega_{\mathrm{i}}=\left\{(x, y):\left(x-d \cos \theta_{0}\right)^{2}+\left(y-d \sin \theta_{0}\right)^{2} \leq r^{2}\right\}$ with $0 \leq \theta_{0} \leq 2 \pi$ and such that $0 \leq r+d \leq 1.0$.

- Case 2 (stratified): $\Omega_{\mathrm{i}}=\left\{(x, y): y \geq r_{0} \cdot \cos \theta\right\}$, with $r_{0}=1$ and $0 \leq \theta \leq \pi$.

- Case 3 (multiple bubbles): a number $n_{b u b} \in\{1,3,11,36,62,84\}$ of uniformly distributed bubbles of radius $r_{d i s}$ is considered. To fix the void fraction relatively to an equivalent concentric bubble of radius $r_{e}$ then $r_{d i s}$ is defined as $r_{d i s}=r_{e} / \sqrt{n_{b u b}}$.

The conductivity distributions of the three cases are depicted in Figure 3 , the area in light blue represents the water phase and the one in white is the gas phase. The electrical conductivity of gas is set to be eight magnitudes lower than that of water, which is in accordance with the physical conductivity values of water and gas. Each case corresponds to specific regimes of two phase flow: in Case 1, one single bubble with various diameter is varied spatially along one specific diameter inside the domain; Case 2 represents stratified flow with various water level, in which $h=1-d=1-\cos \theta$; Case 3 is characterized by multiple bubbles uniformly distributed inside the domain, Figure 3 shows the case with 11 bubbles.
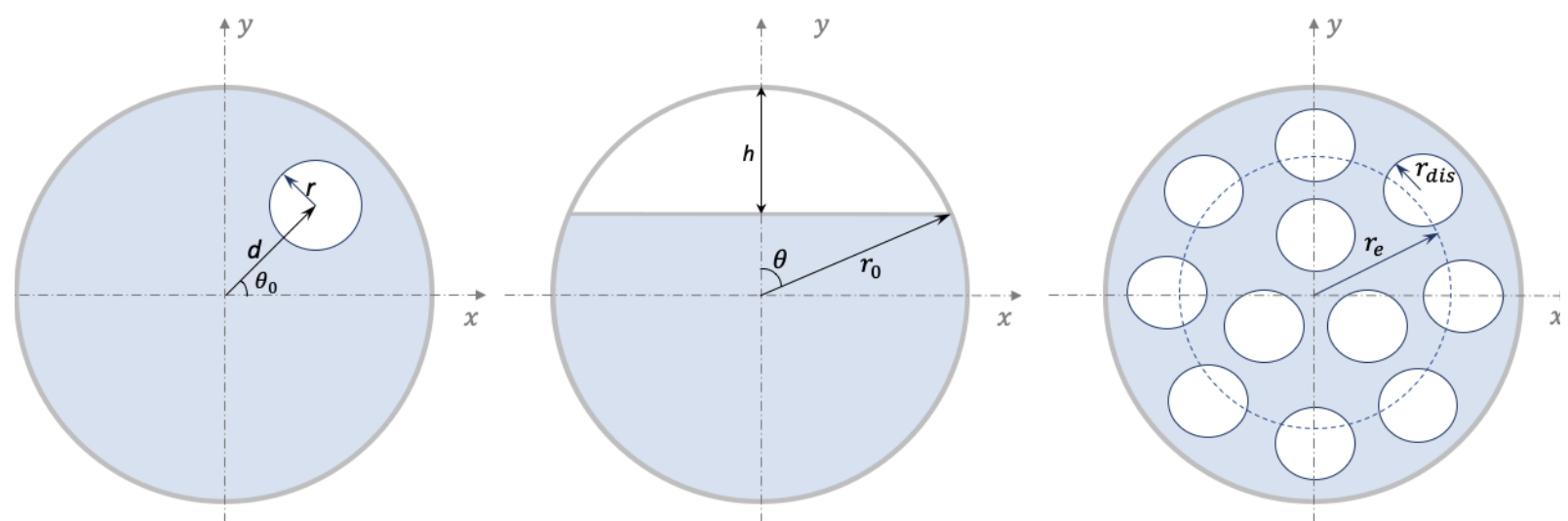

Figure 3. Conductivity distribution patterns for the three canonical cases considered.

To investigate the eigenvalues of the impedance matrix for these three canonical cases, boundary measurements have to be computed. The open source code Electrical Impedance Tomography and Diffuse Optical Tomography Reconstruction Software (EIDORS) is used. It is dedicated to solve EIT forward and inverse problems by the Finite Element Method (FEM) [31].

The numerical model is a $2 \mathrm{D}$ circular model with unit radius and 16 electrodes placed evenly at the boundary, the width of the electrode is chosen to have the ratio between the 
area occupied by the electrodes and the total area the same than the practical EIT system used in our experimental setting. A triangle mesh with 54177 nodes is used in the FEM model.

A full scan stimulation strategy with a constant current of $I_{\text {stimu }}=1 \mathrm{~A}$ at source and drain electrodes is employed. For each stimulation pattern, the difference voltage between adjacent electrodes are computed, denoting the selected source and drain electrodes as $(i, j)$, the voltage difference between source and drain $V_{i, j}$ could be obtained by summing up the voltage differences at all adjacent electrodes between the source and drain. Since the injected current is a constant, the corresponding bulk impedance $Z_{i, j}$ can be obtained by Ohm's law:

$$
Z_{i, j}=V_{i, j} / I_{\text {stimu }} .
$$

With 16 electrodes $(i, j=1, \cdots, 16)$, there are 120 independent stimulation patterns, giving 120 different bulk impedances for one frame of acquisition. After normalization, the square matrix $\hat{Z}$ of size $16 \times 16$ is obtained, along with the $\ell=16$ pairs of eigenvalues and eigenvectors, see Eqn. (12).

The NIM of the three simulated cases considered are visualized to highlight their correlation with the phase pattern. Case 1 is shown in Figure 2(b) with $r=0.3$ and the cases 2 and 3 are shown in Figure 4 , in which Case 2 is computed with $h=0.8$ and Case 3 is computed with 3 bubbles and $r_{e}=0.3$. These parameters are chosen to obtain the same phase fraction for all three cases. As it can be seen, the main features of the NIM vary significantly with the phase pattern, even at the same phase fraction. This characteristic is at the foundation of the present study.

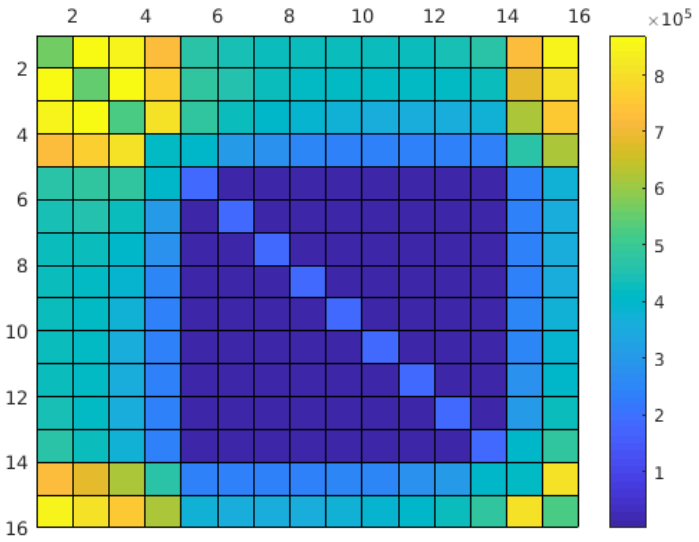

(a) $\hat{Z}$ of case 2 (stratified)

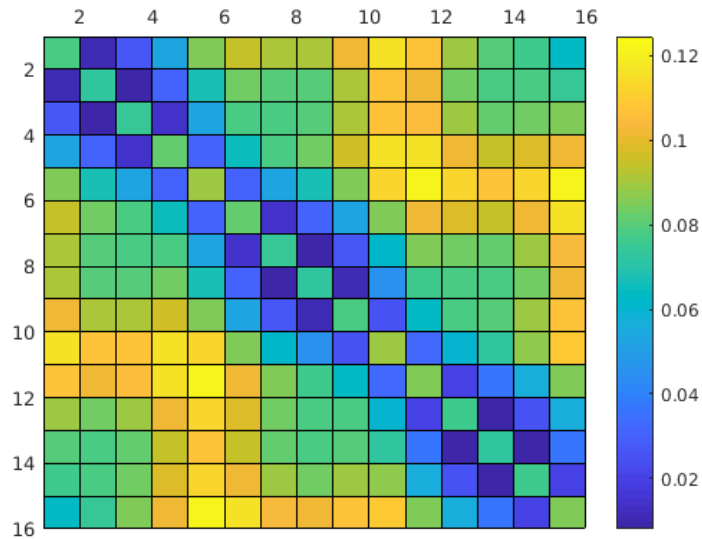

(b) $\hat{\boldsymbol{Z}}$ of case 3 (three bubbles)

Figure 4. Entries of the normalized impedance matrix of the cases 2 and 3.

In the following sections, the eigenvalues of various simulation cases are investigated and compared to study their dependence on the bubble distribution. In particular, the void fraction is plotted with the leading eigenvalue and the sum of the absolute value of the first 15 eigenvalues, to assess their correlations. For convenience, we denote the leading eigenvalue as $\lambda^{(16)}$ and the sum of the absolute value of the first 15 eigenvalues as $\sum_{|\lambda|}^{15}=\left|\lambda^{(1)}\right|+\cdots+\left|\lambda^{(15)}\right|$. 
Moreover, the void fraction $\alpha$ is defined in $2 \mathrm{D}$ by the area ratio occupied by the bubble inclusion over the total area, i.e. $\alpha=\left|\Omega_{\mathrm{i}}\right| /|\Omega|=\left|\Omega_{\mathrm{i}}\right| / \pi$.

\subsection{Result analysis}

\subsubsection{Case 1}

Due to the fact that the properties of the impedance matrix are invariant with the rotation of the electrodes, we only need to simulate the bubble inclusion moving along one radius of the domain. We set $\theta_{0}=0$, which is representative of all cases of a single bubble moving within the domain. Applying the restriction condition $r+d \leq 0.95$ and an increment step of 0.05 , we get 19 different bubble radius $r$ and placements $d$, separately, which will give 190 different simulation cases (190 pairs of $(r, d)$ ) in total. Here, $r+d \leq 0.95$ is chosen because of the extremely high sensitivity near the boundary, which could cause an abnormal deviation, besides, in practical applications it is also rare to have a bubble occupying $95 \%$ of the pipe diameter.

The eigenvalues are computed for each case and plotted. Figure $5(\mathrm{a})$ shows the 16 eigenvalues of concentric bubble inclusions with $d=0$ and $0.05 \leq r \leq 0.95$, while Figure $5(\mathrm{~b})$ shows the 16 eigenvalues of eccentric bubble inclusions with $d=0.35$ and $0.05 \leq r \leq 0.6$. In both figures, each curve represents an eigenvalue $\lambda^{(i)}$, and they are evolving with the bubble radius. As we can see, the leading eigenvalue is much larger than the others, and its amplitude is correlated to the bubble size, especially when the bubble edge is close to the domain boundary. Besides, for concentric bubble configurations, the first 15 eigenvalues are symmetrical around zero due to the symmetry of the conductivity distribution.

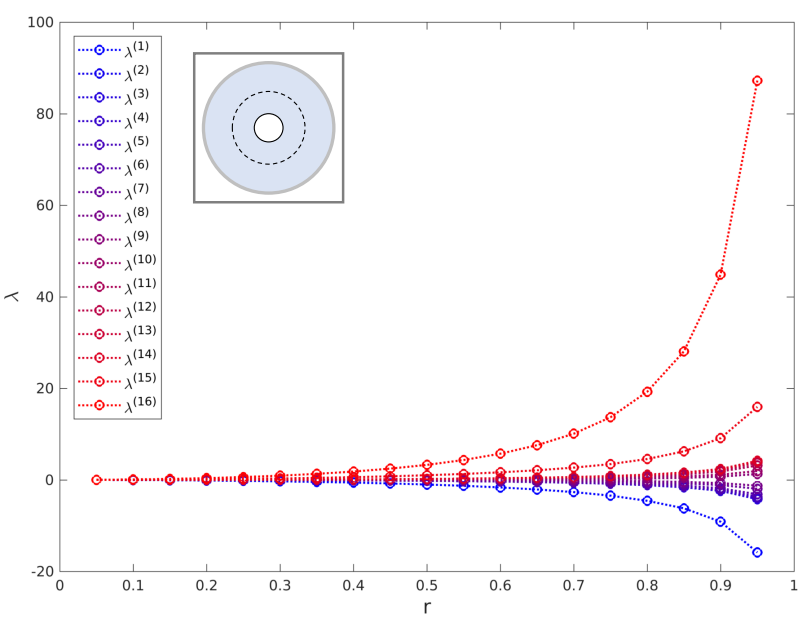

(a) Concentric bubble, $d=0$

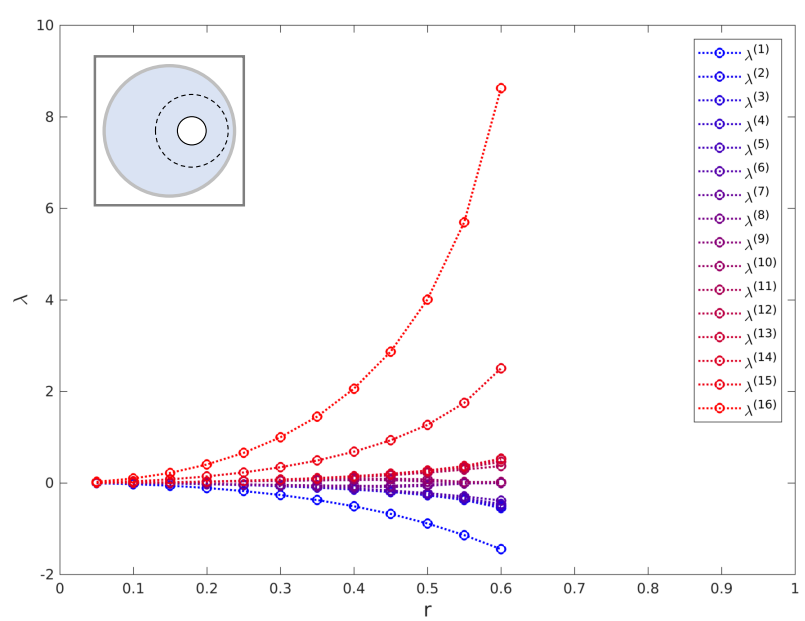

(b) Eccentric bubble, $d=0.35$

Figure 5. Eigenvalue array for some configurations corresponding to Case 1.

Figure 6 shows the 16 eigenvalues of a bubble inclusion with $r=0.35$ moving from $d=0$ to $d=0.6$. As it can be seen, as the bubble moves toward the boundary, the leading eigenvalue increases rapidly, while the other eigenvalues vary little with $d$. 


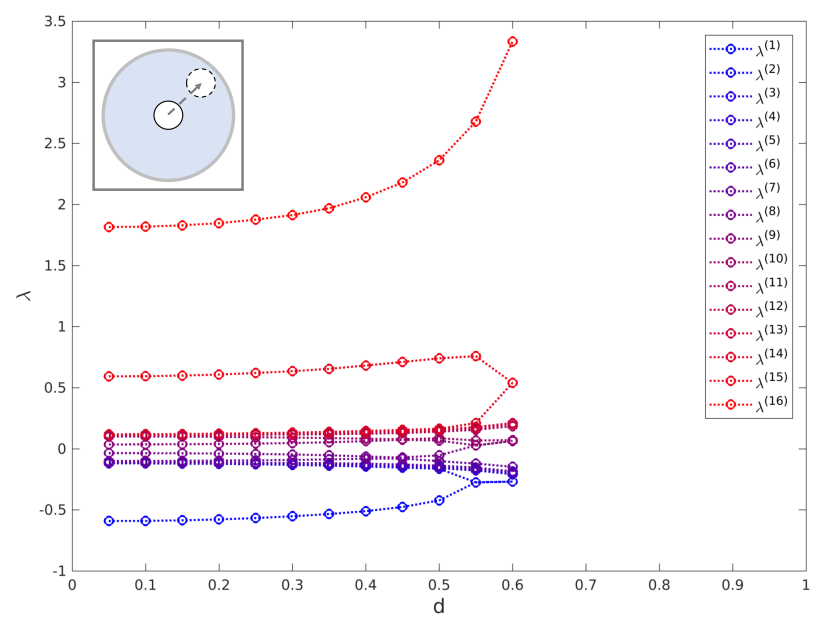

Figure 6. Eigenvalue array of one bubble inclusion $(r=0.35)$ moving from $d=0$ to $d=0.6$.

As we know, adding bubble inclusions increases the overall bulk impedance, hence the normalized impedance $\hat{Z}_{i, j}$, which in turn affects the eigenvalues. That is why the eigenvalues are changing with bubble size. To study the influence of bubble location, the sensitivity map of EIT has to be considered. The sensitivity map $s(x)$ of an EIT sensor quantifies the relation between the change of boundary measurement $\delta V_{\text {meas }}$ caused by a local conductivity perturbation $\delta \sigma(x)$, that is, $s(x)=\delta V_{\text {meas }} / \delta \sigma(x)$. It describes how effectively each region is contributing to the measurement 32 .
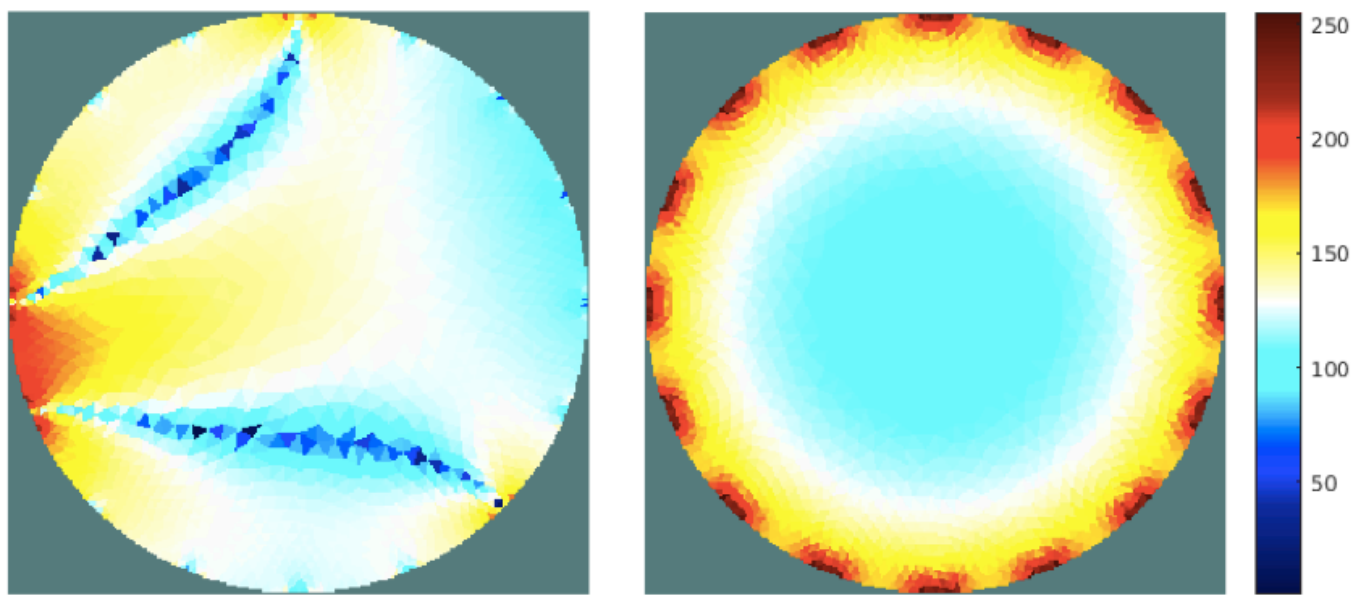

Figure 7. Map of the logarithm of the sensitivity distribution: (left) source/drain at electrodes $(1,7)$ and measurement at electrodes $(12,13)$; (right) full scan strategy.

EIT is a soft-field imaging technique for which high sensitivity areas concentrate near the boundary and the vicinity of the active electrodes while the domain interior contains low sensitivity regions. The sensitivity maps for one single measurement and for the full scan strategy are shown in Figure 7. The color map in the left panel shows the sensitivity distribution of one stimulation pattern with source and drain at electrodes 1 and 7 , and one 
single measurement at electrodes 12 and 13. The sensitivity map of the full scan strategy is shown in the right panel. Considering the same bubble inclusion placed either in the center or near the boundary, the boundary voltage turns out to be more affected on average in the latter configuration, and so are the impedance matrix and its eigenvalues. Thus, the eigenvalues are affected by both the size and position of the bubble inclusion.

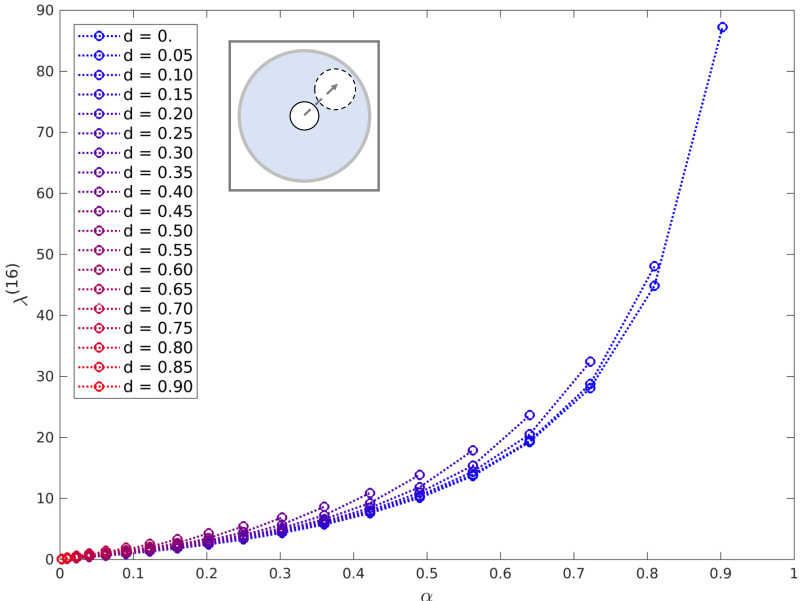

(a) $\lambda^{(16)}$ vs. $\alpha$

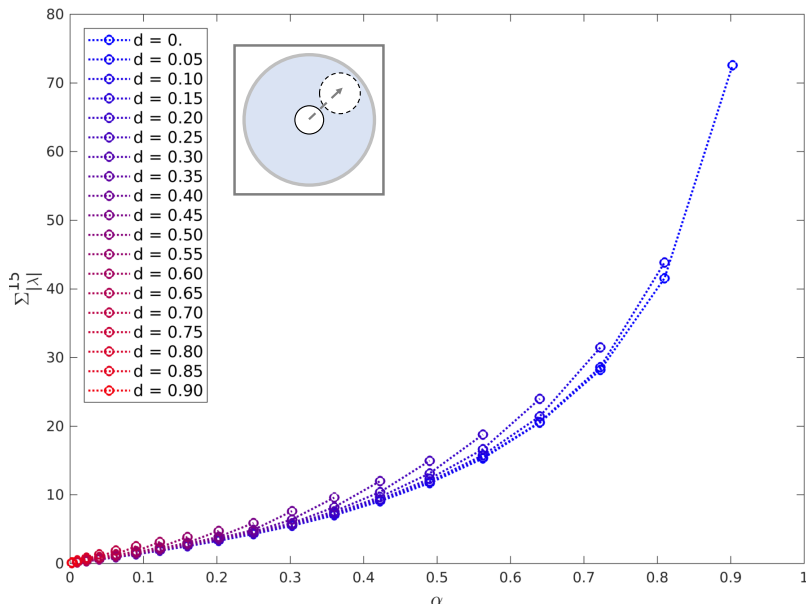

(b) $\sum_{|\lambda|}^{15}$ vs. $\alpha$

Figure 8. Eigenvalues vs. void fraction for Case 1.

Figure 8 shows the relation between the void fraction and the leading eigenvalue $\lambda^{(16)}$ and the sum of the absolute value of the first 15 eigenvalues $\sum_{|\lambda|}^{15}$. For Case 1, the void fraction satisfies $\alpha=\pi r^{2} / \pi r_{0}^{2}=r^{2}$, which is invariant with $d$. In both figures, each curve corresponds to a specific value $d$ with varying $r$, for example, the curve at the right most represents a bubble in the center $(d=0)$, its radius $r$ increases from 0.05 to 0.95 , hence the void fraction ranges from 0 to 0.9025 . The figures show that both $\lambda^{(16)}$ and $\sum_{|\lambda|}^{15}$ increase with the void fraction and vary with the distance to the center. The void fraction can be estimated by $\lambda^{(16)}$ or $\sum_{|\lambda|}^{15}$, from Figure 8 we can estimate the void fraction with an error less than $10 \%$. Furthermore, the error can be reduced drastically when the position of the bubble is known a priori (distance to the center $d$ ).

\subsubsection{Case 2}

In the stratified configuration, a horizontal liquid-gas interface is considered since the impedance matrix is invariant with electrodes rotation. Note that the void fraction $\alpha$ in Case 2 satisfies the relation

$$
\alpha=(\theta-\sin \theta \cdot \cos \theta) / \pi \quad \text { with } \quad \theta=\arccos (1-h),
$$

where $h$ is the thickness of the gas layer as in Figure 3 .

The eigenvalue array of Case 2 is plotted in Figure 9(a). A linear approximation can be found for the relation between $h$ and the leading eigenvalue, while the data points at high void fraction $(h>1.99)$ are considered to be abnormal and discarded. The other eigenvalues are not strongly correlated to $h$. In Figure $9(\mathrm{~b}), \lambda^{(16)}$ is plotted with $h$, and the curve is 
fitted linearly with a fitting measure $R^{2}$ of 0.9905 . The deviation between the fitting line and the data points may originate from the finite number and size of the electrodes and from their limited geometrical extent too.

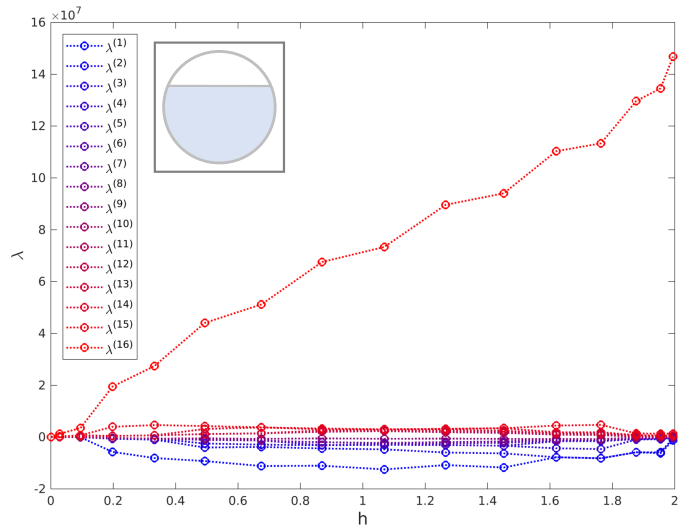

(a) Eigenvalue array vs. $h$

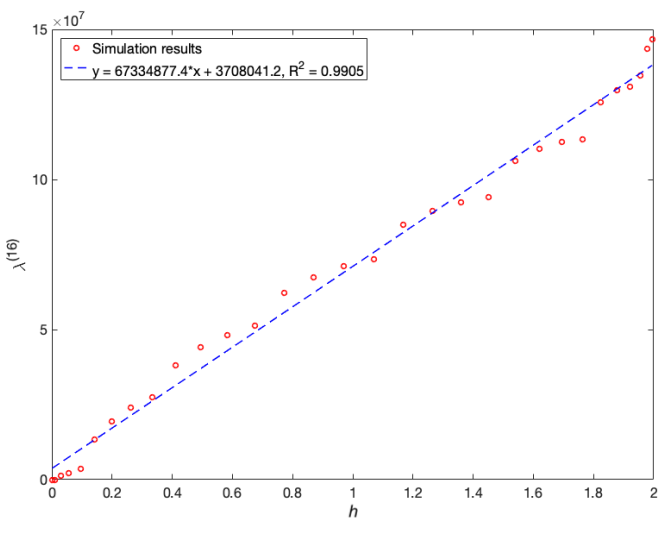

(b) $\lambda^{(16)}$ vs. $h$ and its linear fit

Figure 9. Eigenvalue arrays and $\lambda^{(16)}$ as a function of $h$ for Case 2.

\subsubsection{Case 3}

In Case 3, a number $n_{b u b} \in\{1,3,11,36,62,84\}$ of bubble inclusions are considered with a radius adjusted to get the same water fraction as an equivalent single concentric bubble of radius $r_{e}$. The void fraction of Case 3 is $\alpha=r_{e}^{2}$, which is restricted by $n_{b u b}$ to avoid contact among bubbles. With $n_{b u b}=3$ the top limit of void fraction is 0.5 , for $n_{b u b}=11$ it is 0.6 , while for other cases it ranges from 0.65 to 0.7 . For practical two-phase flows, the intensive bubbly flow rarely reaches a void fraction over 0.5 , otherwise the bubbles would collapse and form bigger bubbles and the flow regime would change.

The eigenvalue $\lambda^{(16)}$ is plotted with the void fraction and the bubbles radius in Figure 10 respectively. In Figure 10(a) the right most curve represents the single concentric bubble case, from right to left the curves correspond to an increasing value of $n_{b u b}$. As we can see, at the same void fraction the leading eigenvalue $\lambda^{(16)}$ is increasing with $n_{b u b}$. The same behaviour is also observed in Figure 10(b): at the same void fraction, $\lambda^{(16)}$ is decreasing with the bubbles radius. Besides, the smaller the bubbles are, the stronger the effect is on $\lambda^{(16)}$, which is shown by the drop of $\lambda^{(16)}$ when $r_{d i s}$ is close to zero. After that point $\lambda^{(16)}$ decreases smoothly as $r_{d i s}$ increases. 


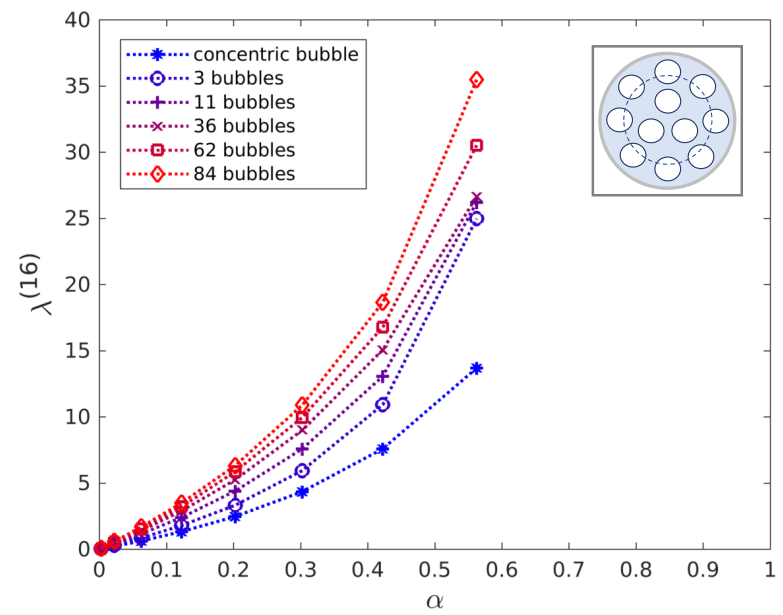

(a) $\lambda^{(16)}$ vs. $\alpha$

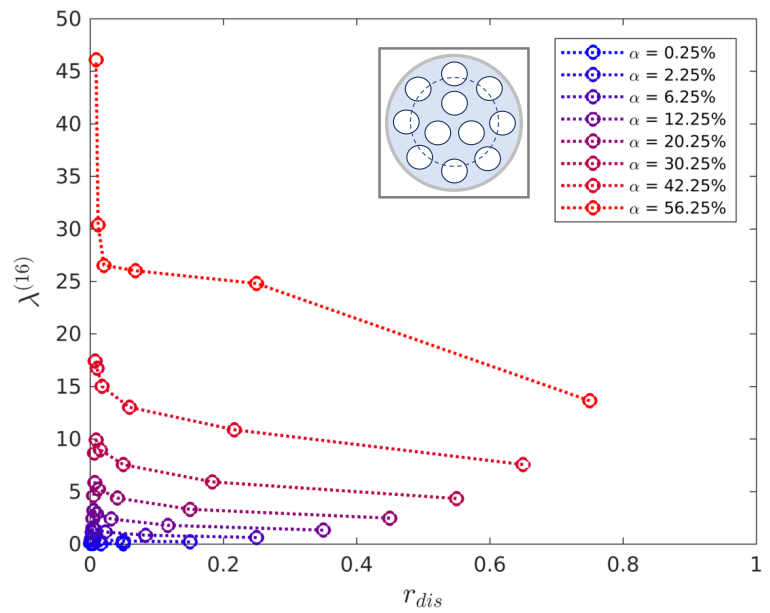

(b) $\lambda^{(16)}$ vs. $r_{e} / \sqrt{n_{b u b}}$

Figure 10. Eigenvalues vs. void fraction for Case 3.

For the multiple bubble case, two parameters have to be known to predict the void fraction: the eigenvalues and the number of bubbles within the sensor area. However, it is not practical to observe the bubble distribution inside a pipe. In the work of Bruhl et al. [33], the eigenvalues of $\Lambda_{\sigma}-\Lambda_{0}$ are used to locate the inhomogeneities and estimate the number of inhomogeneities non-iteratively, which is a very promising method to be combined with the approach proposed here, to obtain a reliable and accurate void fraction estimation for two-phase flows.

\section{Robustness of the proposed approach}

The properties of the eigenvalue distribution of the normalized impedance matrix $\hat{\boldsymbol{Z}}$ are analyzed in the previous section based on 2D simulations. In the present section, the robustness of this methodology is investigated by studying the case where the data is noisy and when the inclusions are conductive.

\subsection{Noisy data}

In practical EIT systems, the boundary measurements are always polluted by some noise, including background white noise or the cross-talk of electrical devices. Thus, the robustness of the eigenvalue analysis to noisy data has to be assessed. The Signal-to-Noise Ratio (SNR) of the EIT system developed for the present study at the Laboratory of Thermal-Hydraulics in Core and Circuits (LTHC) is higher than $60 \mathrm{~dB}$ for all channels at a stimulation frequency of $20 \mathrm{kHz}$ and amplitude of $1 \mathrm{~V}$. Given this SNR value then adding a noise of $10 \mathrm{~dB}$ to the measurement data from numerical simulations is conservative.

The noise is added to the measurement data from both the homogenous and inhomogeneous configurations. The eigenvalues of Case 1 are computed and compared to the results without noise. Again, $\lambda^{(16)}$ and $\sum_{|\lambda|}^{15}$ are the metrics this study is focusing on, so they are plot- 


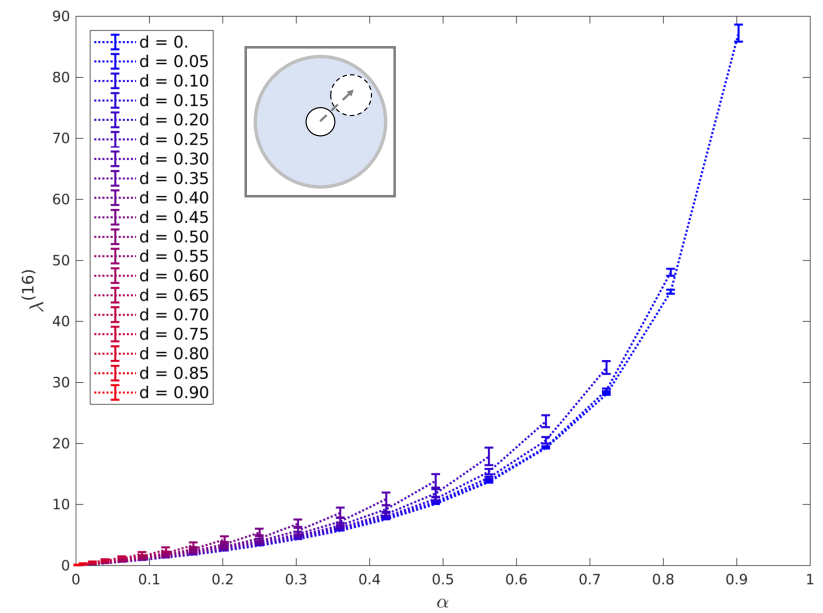

(a) $\lambda^{(16)}$ vs. $\alpha$ with error bars

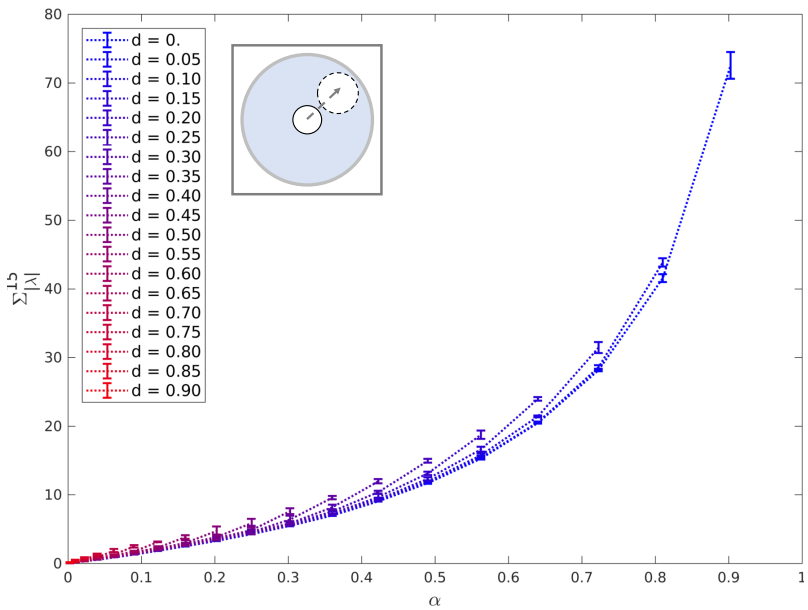

(b) $\sum_{|\lambda|}^{15}$ vs. $\alpha$ with error bars

Figure 11. Eigenvalue trends obtained with noisy data in Case 1.

ted with error bars as in Figure 11. The error $\xi$ on $\lambda^{(16)}$ is computed as $\xi=\left|\lambda_{\text {noisy }}^{(16)}-\lambda^{(16)}\right| / \lambda^{(16)}$ where $\lambda^{(16)}$ is computed without noise and $\lambda_{\text {noisy }}^{(16)}$ is computed with a 10dB noise. The error on $\sum_{|\lambda|}^{15}$ is computed in the same fashion. As we can see, the relative error for both $\lambda^{(16)}$ and $\sum_{|\lambda|}^{15}$ are very small, the maximum values being $4.3 \%$ and $2.0 \%$ respectively.

\subsection{Extension to conductive inclusions}

Apart from the application in water-gas two phase flow measurements, EIT sensors can also be used in various industrial processes, which may concern conductive inclusions rather than non-conductive bubbles. Here, the definition of the conductivity contrast $\mathcal{R}=\sigma_{\text {high }} / \sigma_{\text {low }}$ from Seagar et al. [34] is used to represent the conductivity difference between two phases. For water-gas two-phase flows, $\mathcal{R}$ tends to infinity. In the section, some configurations with $\mathcal{R} \in\{100,10,5,2.5,1.25\}$ are simulated and compared to the results associated with $\mathcal{R} \approx \infty$ (i.e. water-gas two-phase flows), to assess the general applicability of the proposed methodology.

All three cases are simulated in 2D when varying $\mathcal{R}$ and the results for the cases 1 and 2 are plotted in Figures 12 to 13 . In Figure 12(a), the eigenvalue $\lambda^{(16)}$ for the single concentric bubble case is plotted with $\alpha$ and each curve represents different values of $\mathcal{R}$. As we can see, the trends of $\lambda^{(16)}$ changing with $\alpha$ are similar for different values of $\mathcal{R}$, while the amplitude are increasing globally with $\mathcal{R}$. Also, as $\mathcal{R}$ increases to large values, $\lambda^{(16)}$ increases less and less, especially after $\mathcal{R}=100$, i.e. there exists a threshold effect. The same conclusions are obtained for $\sum_{|\lambda|}^{15}$, see Figure $12(\mathrm{~b})$.

For the stratified case, the same results can be obtained, while the contrast $\mathcal{R}$ has a much stronger effect on the eigenvalues, especially for large values. As shown in Figure $13, \lambda^{(16)}$ is 5 to 6 magnitudes higher for $\mathcal{R}=\infty$ than for the other cases, so that $\lambda^{(16)}$ vs. $h$ is plotted in Figure 13(b) excluding the case $\mathcal{R}=\infty$. Nevertheless, a linear correlation can be found between $\lambda^{(16)}$ and $h$ for varying $\mathcal{R}$. 


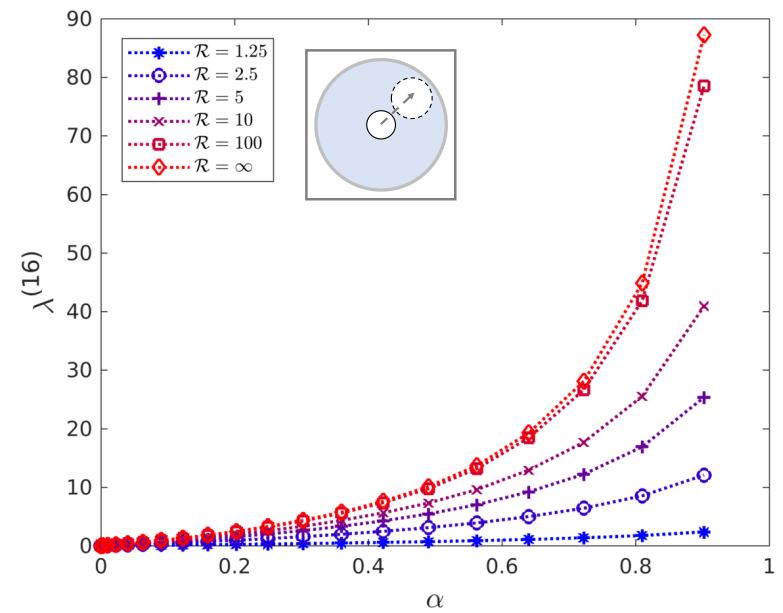

(a) $\lambda^{(16)}$ vs. $\alpha$

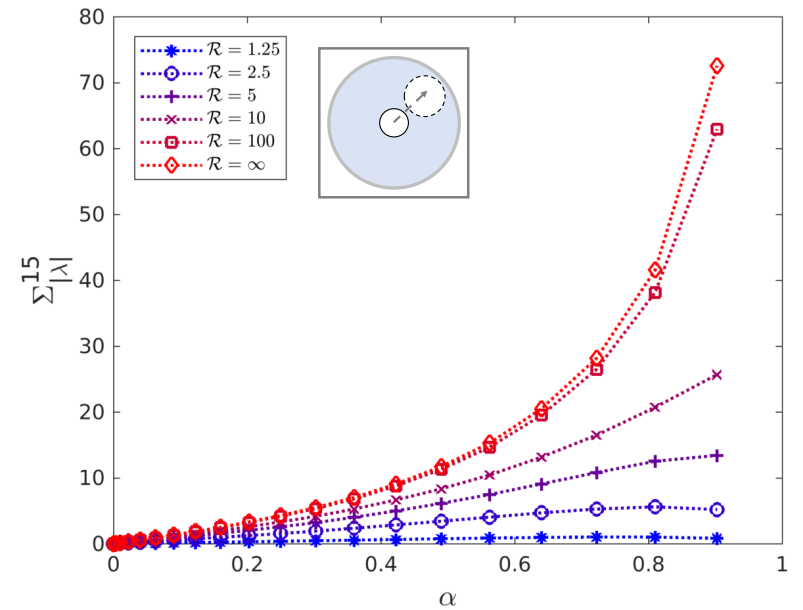

(b) $\sum_{|\lambda|}^{15}$ vs. $\alpha$

Figure 12. $\lambda^{(16)}$ and $\sum_{|\lambda|}^{15}$ vs. $\alpha$ for various values of $\mathcal{R}$ in Case 1.

Lastly, the results for the multiple bubble case have been found to be similar to the single bubble case. The applicability of the eigenvalue analysis to conductive inclusions is therefore emphasized but the conductivity contrast $\mathcal{R}$ between phases is an important information to be known beforehand.

\section{$5 \quad$ Validation by experimental static tests}

In this section, the methodology for void fraction estimation, which has been devised based on numerical simulations, is validated experimentally. We perform static tests, with a pipe containing still water, for a number of configurations corresponding to the three inclusion patterns considered previously. The motivations for performing such static tests are as follows: (i) they are easily implementable using standard laboratory equipment and allow to control the phase distribution accurately, which is crucial to validate the proposed approach, (ii) EIT measurements can be performed at a high frame rate (of the order of 800fps for the system considered), which allows to treat a dynamic flow as if it were quasi-static on each frame. These experimental tests are carried out with the EIT system developed in LTHC.

\subsection{EIT system in LTHC}

The prototype EIT sensor has 16 electrodes on the boundary of the test section with an angular separation of $22.5^{\circ}$. The test section is a pipe with diameter of $80 \mathrm{~mm}$ and height of $300 \mathrm{~mm}$ while the electrode size is $170 \mathrm{~mm} \times 5 \mathrm{~mm}$ [9], as shown in Figure 14 . 


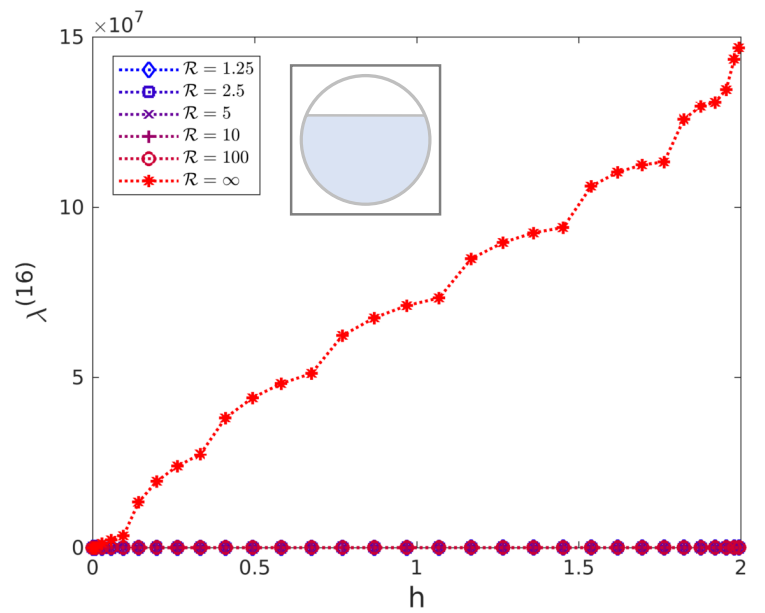

(a) $\lambda^{(16)}$ vs. $h$, all cases

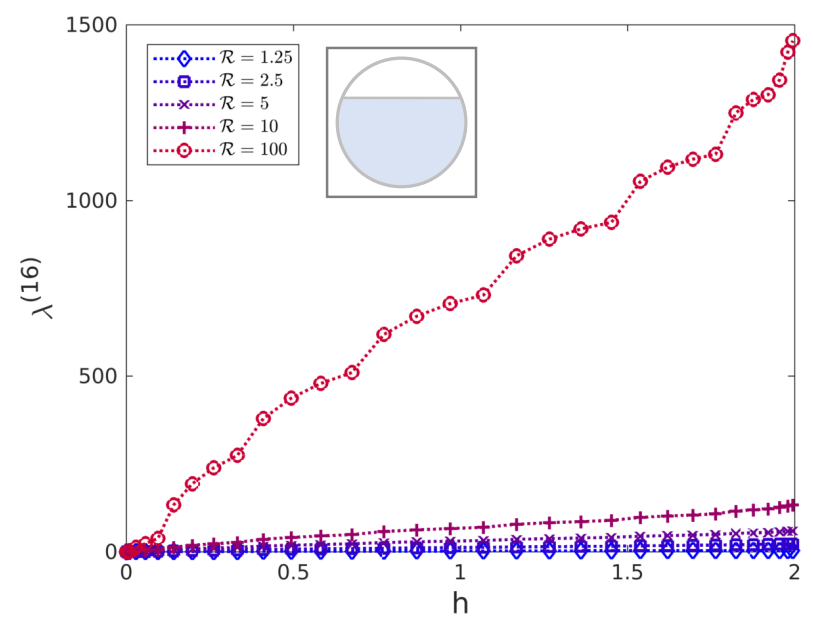

(b) $\lambda^{(16)}$ vs. $h$, excluding $\mathcal{R}=\infty$

Figure 13. $\lambda^{(16)}$ vs. $h$ in Case 2.

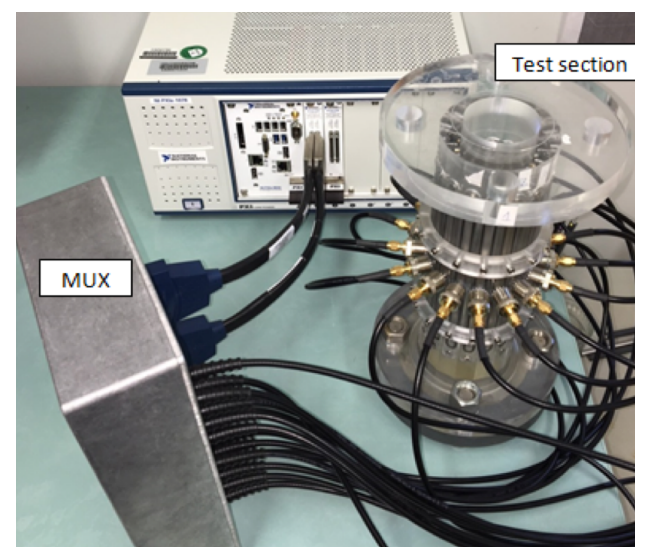

Figure 14. EIT system in LTHC.

Stimulation and signal acquisition are both performed using the PXIe system from National Instrument. The stimulation signal is a sinuous voltage with amplitude of $1 \mathrm{~V}$ to avoid the electrolysis reaction of water. The signal is generated by the analogue output channel of the PXIe system, the sample rate ranges from $1 \mathrm{~Hz}$ to $3.33 \mathrm{MHz}$. Data acquisition is done by the differential analogue input channels of the system, the simultaneous sample rate of all channels reaches up to $2 \mathrm{MHz}$ [35]. A multiplexer with 16 ports is used to route the stimulation signal to a selected pair of electrodes. A discrete Fourier transform is performed on the measurement data to obtain amplitudes and phases at the stimulation frequency.

Static tests are carried out with still water filling the test section and a number of plastic rods emulating bubbles. Different diameters and numbers of rods are placed into the water to get various void fractions. In a first step, the impedance of each stimulation pattern has to be obtained from practical measurements. The prototype EIT uses voltage of $\pm 1 V$ as a stimulation signal, which is different from the current stimulation considered in the numerical simulations but we consider the two settings as being equivalent. Each stimulation pattern 
can be assumed as a closed circuit as in Figure 15, in which the bulk impedance $Z^{\text {bulk }}$ and the contact impedances at the source/drain electrodes $Z^{\text {source }}$ and $Z^{\text {drain }}$ are connected as

$$
Z^{\text {tot }}=Z^{\text {source }}+Z^{\text {drain }}+Z^{\text {bulk }}=V / I \text {. }
$$

The terms $Z^{\text {source }}$ and $Z^{\text {drain }}$ are essentially equal, they are electrode-specific and invariant with phase distributions. In (15), $V$ is the stimulation voltage and $I$ is the sensor current.

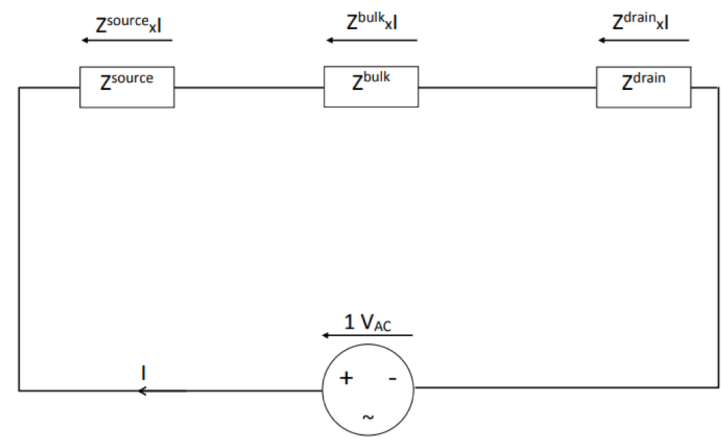

Figure 15. Closed circuit between the source and drain electrodes.

In the EIT system, a constant resistor $R_{0}=200 \Omega$ is included in the closed circuit and the voltage difference $\Delta V_{R_{0}}$ across $R_{0}$ is measured. Thus, the current $I$ through the circuit can be obtained as $I=\Delta V_{R_{0}} / R_{0}$. Considering the homogeneous case, there are 120 independent equations as Eqn. (15). As $Z^{\text {bulk }}$ depends only on the angular separation of source/drain electrodes, there are 8 different values of $Z^{\text {bulk }}$ overall. Together with the 16 different values of $Z^{\text {source }}$ ( or $Z^{\text {drain }}$ ), the set of 120 linear equations contains 24 unknown parameters. Thus the values of $Z^{\text {source }}$ and $Z^{\text {drain }}$ can be obtained and used to apply the proposed methodology. For inhomogeneous configurations, the bulk impedances are computed as in the homogeneous case.

\subsection{Result comparison}

The static test results are compared to numerical simulations for validation. Simulations in 2D can be considered as being equivalent to configurations with infinite long electrodes and inclusions, while in practical 3D implementations, the electrodes and the inclusions have finite extents. Therefore, it is more relevant to compare the results from a practical EIT system with 3D simulations. As a consequence, we consider here a 3D cylindrical model implemented in EIDORS. The radius of the numerical model is 1 while all other parameters are normalized from the prototype EIT system so that the length of the model is 7.5 , the electrodes have a width of 0.125 and a length of 4.25 . The bubble inclusion is emulated by a non-conductive rod of the height of the model. The 3D numerical model has 198730 tetrahedral mesh elements.

As of the experimental tests, a series of configurations are investigated to obtain data for all of the three patterns considered. For Case 1, a single non-conductive rod with different diameters is placed in the test section at different distances to the center: the diameter of the 


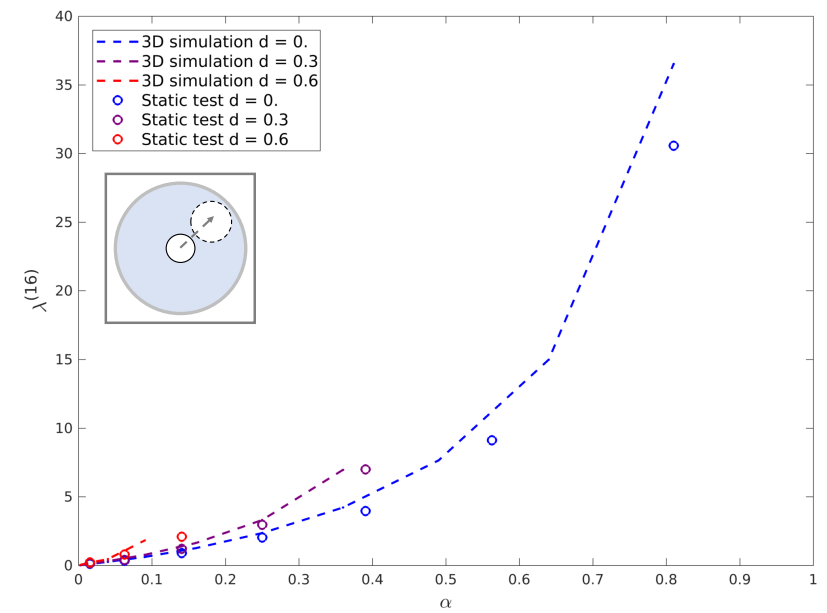

(a) Case 1

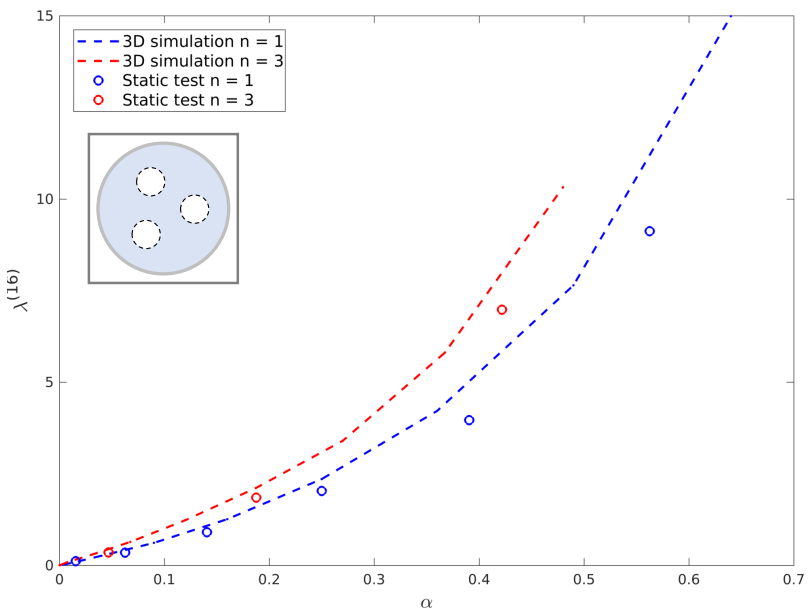

(b) Case 3

Figure 16. Comparison between 3D simulations and experimental static tests for the cases 1 and 3: eigenvalue $\lambda^{(16)}$ as a function of the void fraction $\alpha$.

rod ranges from $10 \mathrm{~mm}$ to $70 \mathrm{~mm}$, the distance to the center being $d \in\{0,12 \mathrm{~mm}, 24 \mathrm{~mm}\}$, corresponding to $d \in\left\{0,0.3 r_{0}, 0.6 r_{0}\right\}$ in the $3 \mathrm{D}$ simulations. For Case 2, the test section is placed horizontally with different water levels. In Case 3, different tests with three rods placed symmetrically are carried out, the diameter of the rods ranges from $10 \mathrm{~mm}$ to $40 \mathrm{~mm}$. The normalized impedance matrix and its eigenvalues are computed for each test. The evolutions of the eigenvalue $\lambda^{(16)}$ with the void fraction are shown along with 3D simulation results in Figure 16 for the cases 1 and 3, and in Figure 17 for Case 2.

For the cases 1 and 3 , the trends of the eigenvalue $\lambda^{(16)}$ for the static tests agree well with the $3 \mathrm{D}$ simulations, which validates the proposed methodology. Deviations of low relative amplitude can be observed, which may be due to the following facts: (i) the simulation model is non-dimensional with normalized size and conductivity; (ii) the stimulation signal is a constant current in the simulations while it is a constant voltage in the experiments.

For Case 2, the trends of the eigenvalue $\lambda^{(16)}$ with the thickness $h$ of the gas layer, see (14), for the experiments and the 3D numerical simulations are very different, as seen in Figure $17(\mathrm{a})$, especially at small $h$. The magnitudes in the experiments are also significantly lower than in the simulations. These discrepancies may come from: (i) the surface tension of water causes a rise at the interface between the plastic pipe and the water, especially at low $h$; (ii) a conductive water film may be present on the uncovered part of the pipe surface, while in the simulation there is no such film. Moreover, the magnitude of the eigenvalue $\lambda^{(16)}$ is remarkably high for all values of $h$ compared to the other phase distribution patterns (at the same void fraction), as shown in Figure 17(b), Lastly, the electrodes that are immersed in water can be directly revealed by the distribution of the entries of the normalized impedance matrix, as seen in Figure 4(a), which could therefore be used as an indicator of the water level. 


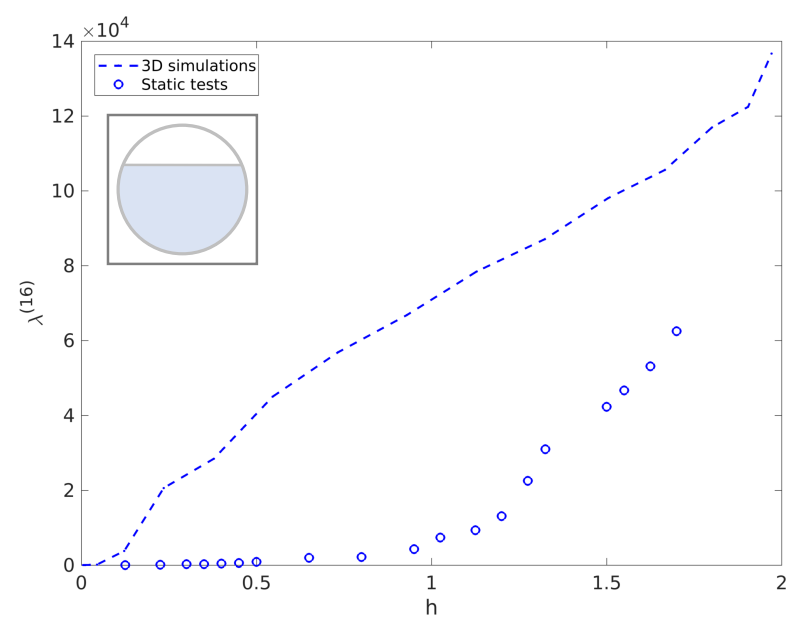

(a) $\lambda^{(16)}$ vs. $h$

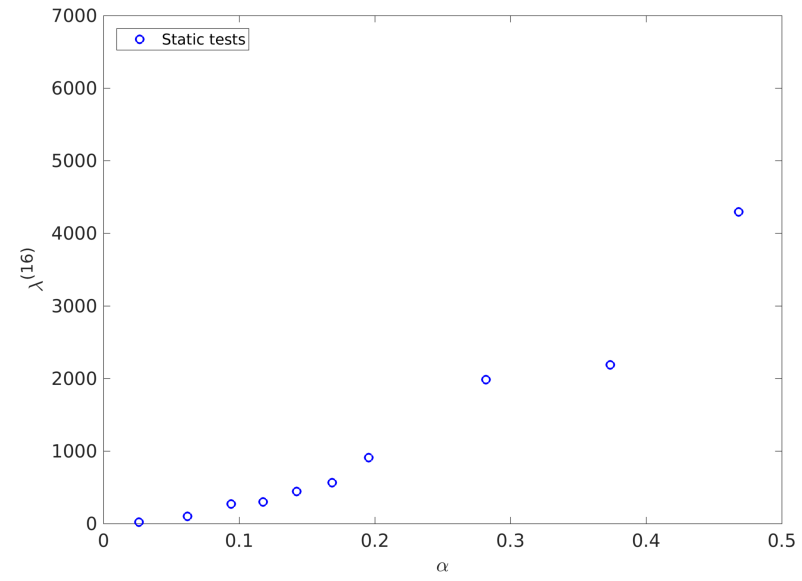

(b) $\lambda^{(16)}$ vs. $\alpha$ for low values of $h$

Figure 17. Comparison between 3D simulations and experimental static tests for Case 2.

\section{Conclusion}

In this article, the impedance data from electrical impedance tomography sensor and its relation to the void fraction of two-phase flows is investigated numerically and experimentally for a cylindrical pipe configuration. The forward problem associated with EIT is a boundary value problem: prescribing Neumann boundary conditions, the Dirichlet boundary measurements depend on the internal conductivity distribution. In practical implementations, discrete electrodes are used for stimulation and boundary measurements, leading to one bulk impedance matrix for each stimulation pattern. A normalization of the impedance matrix is considered to extract information from the data and reduce the influences of factors other than the bubble inclusion distribution, such as the diameter of the sensor, the background medium conductivity or the stimulation signal.

Numerical simulations are carried out for three different canonical cases that cover a diversity of bubble distribution patterns for two-phase flows. Synthetic boundary measurements are computed to obtain normalized impedance matrices, the eigenvalues of which are investigated in the different configurations considered. The robustness of the proposed approach to noisy data is assessed by adding $10 \mathrm{~dB}$ of noise to the measurement data and the presented results highlight some satisfying performances. Moreover, this methodology can be extended to case of conductive inclusions.

From the numerical simulations, it is found that the leading eigenvalue $\lambda^{(16)}$ and the sum of eigenvalues $\sum_{|\lambda|}^{15}=\left|\lambda^{(1)}\right|+\cdots+\left|\lambda^{(15)}\right|$ are strongly correlated to the void fraction for all of the cases considered: (i) in the single bubble case (Case 1), the simulation results are encapsulated by the curves of $\lambda^{(16)}$ and $\sum_{|\lambda|}^{15}$ vs. $\alpha$ excluding the data point at $\alpha>0.9025$. The void fraction can be estimated by $\sum_{|\lambda|}^{15}$ with an error of $10 \%$ regardless of the bubble position; (ii) in the stratified case (Case 2), a linear correlation is found between $\lambda^{(16)}$ and $h$ with a fitting measure $R^{2}=0.9905$; (iii) in the multiple bubble case (Case 3 ), the relation between the void fraction and the eigenvalues are strongly dependent on the number $n_{b u b}$ 
of inclusions and an accurate estimation of the void fraction requires the knowledge of both $\lambda^{(16)}$, or $\sum_{|\lambda|}^{15}$, and $n_{b u b}$.

Experimental static tests corresponding to the three cases considered are carried out and the eigenvalues of the NIM from experimental data are compared with 3D simulations. The trends for the eigenvalue $\lambda^{(16)}$ with $\alpha$ agree well for the cases 1 and 3, although there are small deviations in terms of the magnitudes; for Case 2 there is a large discrepancy between the static tests and the simulations, while $\lambda^{(16)}$ is remarkably high at all $h$ compared to other phase distribution patterns at the same phase fraction.

For a given EIT sensor with specific electrode size and placement, the void fraction can be estimated from the eigenvalues of the NIM. The estimation error would be reduced by incorporating a priori knowledge on the flow regime, which can be identified too from EIT data, see 19. This overall approach provides a good estimation of the void fraction in twophase flows without performing image reconstruction, especially for annular flow (concentric bubble column) and stratified flow. While the eigenvalue-based indicators considered here constitute valuable metrics for the state of the system, there is a need for an in-depth mathematical analysis of the relation between the system and the eigenvalues of the impedance matrix. Such an analysis would allow to determine which information can be retrieved from such a matrix and which metrics are suitable to do so. Lastly and as in [36], this methodology can be used to devise an initial guess for iterative image reconstruction algorithms thereby improving their convergence. For further improvements, the MUltiple SIgnal Classification (MUSIC) algorithm, see [33, could be employed to obtain the number and positions of bubble inclusions within the probed medium to improve the estimation of the void fraction.

\section{References}

[1] Neil E Todreas and Mujid S Kazimi. Nuclear Systems Volume I: Thermal Hydraulic Fundamentals. CRC press, 2011.

[2] Guillaume Ricciardi, M Pettigrew, and Njuki Mureithi. Fluidelastic instability in a normal triangular tube bundle subjected to air-water cross-flow. Journal of Pressure Vessel Technology, 133:9, 102011.

[3] HF Velasco Peña and OMH Rodriguez. Applications of wire-mesh sensors in multiphase flows. Flow measurement and instrumentation, 45:255-273, 2015.

[4] J Enrique Julia, Wouter K Harteveld, Robert F Mudde, and Harrie EA Van den Akker. On the accuracy of the void fraction measurements using optical probes in bubbly flows. Review of scientific instruments, 76(3):035103, 2005.

[5] Owen C Jones Jr and Jean-Marc Delhaye. Transient and statistical measurement techniques for two-phase flows: a critical review. International Journal of Multiphase Flow, 3(2):89-116, 1976.

[6] Theodore J Heindel. A review of x-ray flow visualization with applications to multiphase flows. Journal of Fluids Engineering, 133(7):074001, 2011.

[7] César Marques Salgado, Cláudio MNA Pereira, Roberto Schirru, and Luis EB Brandão. Flow regime identification and volume fraction prediction in multiphase flows by means of gamma- 
ray attenuation and artificial neural networks. Progress in Nuclear Energy, 52(6):555-562, 2010 .

[8] Zhiyao Huang, Baoliang Wang, and Haiqing Li. Application of electrical capacitance tomography to the void fraction measurement of two-phase flow. IEEE Transactions on Instrumentation and Measurement, 52(1):7-12, 2003.

[9] Antoine Dupré, Guillaume Ricciardi, and Salah Bourennane. Novel approach for analysis and design of high-speed electrical impedance tomographic system for void fraction measurements in fast two-phase flows. IEEE Sensors Journal, 17(14):4472-4482, 2017.

[10] Erkki Somersalo, Margaret Cheney, and David Isaacson. Existence and uniqueness for electrode models for electric current computed tomography. SIAM Journal on Applied Mathemat$i c s, 52(4): 1023-1040,1992$.

[11] Fadil Santosa and Michael Vogelius. A backprojection algorithm for electrical impedance imaging. SIAM Journal on Applied Mathematics, 50(1):216-243, 1990.

[12] Margaret Cheney, David Isaacson, Jonathan C Newell, S Simske, and J Goble. Noser: An algorithm for solving the inverse conductivity problem. International Journal of Imaging Systems and Technology, 2(2):66-75, 1990.

[13] Luiz Felipe Fuks, Margaret Cheney, David Isaacson, David G Gisser, and JC Newell. Detection and imaging of electric conductivity and permittivity at low frequency. IEEE Transactions on Biomedical Engineering, 38(11):1106-1110, 1991.

[14] Alberto P Calderón. On an inverse boundary value problem. Computational \& Applied Mathematics, 25(2-3):133-138, 2006.

[15] Robert V Kohn and Alan McKenney. Numerical implementation of a variational method for electrical impedance tomography. Inverse Problems, 6(3):389, 1990.

[16] Thomas J Yorkey, John G Webster, and Willis J Tompkins. Comparing reconstruction algorithms for electrical impedance tomography. IEEE Transactions on Biomedical Engineering, BME-34(11):843-852, 1987.

[17] Liliana Borcea. Electrical impedance tomography. Inverse problems, 18(6):R99, 2002.

[18] Margaret Cheney, David Isaacson, and Jonathan C Newell. Electrical impedance tomography. SIAM review, 41(1):85-101, 1999.

[19] Antoine Dupre. Electrical impedance tomography for void fraction measurements of harsh twophase flows: prototype development and reconstruction techniques. PhD thesis, Ecole Centrale Marseille, 2017.

[20] Weifu Fang and Ellis Cumberbatch. Matrix properties of data from electrical capacitance tomography. Journal of engineering mathematics, 51(2):127-146, 2005.

[21] Antoine Dupré, Guillaume Ricciardi, Salah Bourennane, and Saba Mylvaganam. Electrical capacitance-based flow regimes identification - multiphase experiments and sensor modeling. IEEE Sensors Journal, 17(24):8117-8128, 2017. 
[22] Chunhui Dang, Mathieu Darnajou, Guillaume Ricciardi, Achim Beisiegel, Salah Bourennane, and Cédric Bellis. Performance analysis of an electrical impedance tomography sensor with two sets of electrodes of different sizes. In Proceedings of WCIPT-9, Bath, UK, 2018.

[23] Martin Hanke and Martin Brühl. Recent progress in electrical impedance tomography. Inverse Problems, 19(6):S65, 2003.

[24] Kuo-Sheng Cheng, David Isaacson, JC Newell, and David G Gisser. Electrode models for electric current computed tomography. IEEE Transactions on Biomedical Engineering, 36(9):918$924,1989$.

[25] M Wang. Electrode models in electrical impedance tomography. Journal of Zhejiang University-SCIENCE A, 6(12):1386-1393, 2005.

[26] SL Ceccio and DL George. A review of electrical impedance techniques for the measurement of multiphase flows. Journal of fluids engineering, 118(2):391-399, 1996.

[27] DL George, JR Torczynski, KA Shollenberger, TJ O?Hern, and SL Ceccio. Validation of electrical-impedance tomography for measurements of material distribution in two-phase flows. International Journal of Multiphase Flow, 26(4):549-581, 2000.

[28] Yemada Taitel and Abe E Dukler. A model for predicting flow regime transitions in horizontal and near horizontal gas-liquid flow. AIChE journal, 22(1):47-55, 1976.

[29] L Rossi, R De Fayard, and S Kassab. Measurements using x-ray attenuation vertical distribution of the void fraction for different flow regimes in a horizontal pipe. Nuclear Engineering and Design, 336:129-140, 2018.

[30] Geoffrey Frederick Hewitt and DN Roberts. Studies of two-phase flow patterns by simultaneous x-ray and flast photography. Technical report, Atomic Energy Research Establishment, Harwell, England (United Kingdom), 1969.

[31] Nick Polydorides and William RB Lionheart. A matlab toolkit for three-dimensional electrical impedance tomography: a contribution to the electrical impedance and diffuse optical reconstruction software project. Measurement science and technology, 13(12):1871, 2002.

[32] P Kauppinen, J Hyttinen, and J Malmivuo. Sensitivity distribution simulations of impedance tomography electrode combinations. In BEM \& NFSI Conference Proceedings, volume 7, pages 344-347, 2005.

[33] Martin Brühl. Explicit characterization of inclusions in electrical impedance tomography. SIAM Journal on Mathematical Analysis, 32(6):1327-1341, 2001.

[34] AD Seagar, DC Barber, and BH Brown. Theoretical limits to sensitivity and resolution in impedance imaging. Clinical Physics and Physiological Measurement, 8(4A):13, 1987.

[35] NI PXIe. DEVICE SPECIFICATIONS NI 6368 X Series Data Acquisition. National Instrument, 2016.

[36] Cédric Bellis, Andrei Constantinescu, Thomas Coquet, Thomas Jaravel, and Armin Lechleiter. A non-iterative sampling approach using noise subspace projection for EIT. Inverse Problems, 28(7):075015, 2012. 